(C)2012 American Physical Society. Access to this work was provided by the University of Maryland, Baltimore County (UMBC) ScholarWorks@UMBC digital repository on the Maryland Shared Open Access (MD-SOAR) platform.

Please provide feedback

Please support the ScholarWorks@UMBC repository by emailing scholarworks-group@umbc.edu and telling us

what having access to this work means to you and why it's important to you. Thank you. 


\title{
Time-bin-entangled photon holes
}

\author{
J. Liang, " J. D. Franson, and T. B. Pittman \\ Physics Department, University of Maryland Baltimore County, Baltimore, Maryland 21250, USA
}

(Received 23 August 2012; published 28 November 2012)

\begin{abstract}
The general concept of entangled photon holes is based on a correlated absence of photon pairs in an otherwise constant optical background. Here we consider the specialized case when this background is confined to two well-defined time bins, which allows the formation of time-bin-entangled photon holes. We show that when the typical coherent-state background is replaced by a true single-photon (Fock state) background, the basic time-bin-entangled photon-hole state becomes equivalent to one of the time-bin-entangled photon-pair states. We experimentally demonstrate these ideas using a parametric down-conversion photon-pair source, linear optics, and postselection to violate a Bell inequality with time-bin-entangled photon holes.
\end{abstract}

DOI: 10.1103/PhysRevA.86.053831

PACS number(s): 42.65.Lm, 03.67.Bg, 42.50.Dv, 42.50.St

\section{INTRODUCTION}

Entangled photon-hole (EPH) states are a new form of entanglement that is based on the existence of "missing pairs" of photons in two optical modes [1]. These states have now been observed in the laboratory [2], and their nonclassical properties have been experimentally demonstrated [3]. It has recently been shown that these states can be relatively insensitive to loss and amplification noise [4], which may be beneficial for quantum communication applications such as quantum key distribution [5].

All of these initial studies [1-4,6] have only considered a kind of energy-time EPH state [7] where, roughly speaking, the time scale associated with the correlated absences (i.e., the "photon holes") is much shorter than the coherence time of the background states in which they exist. Motivated by the many different forms of entangled photon-pair states (e.g., polarization entanglement $[8,9]$, momentum entanglement [10], etc.), this naturally raises the question of what other forms of EPH states might look like. Along these lines, in this paper we introduce the idea of time-bin EPH states, in analogy with time-bin-entangled photon-pair states pioneered by Gisin's group [11,12].

The initial EPH studies [1-4,6] have also only considered the case where the background in which the photon holes exist is formed by coherent states. This raises questions about the possibility of photon holes in different types of background states, such as thermal states or even various nonclassical states. Here we consider time-bin EPHs in a background formed by highly nonclassical single-photon states.

The combination of time bins and a single-photon background is particularly useful for illustrating the concept of EPHs by comparison and contrast with more familiar entangled photon-pair states. Indeed, as a coherent-state background is replaced by a single-photon-state background, a basic timebin EPH state becomes fully equivalent to an antisymmetric time-bin-entangled photon-pair state. This relatively simple scenario provides insight into the properties of more general EPH states, such as energy-time EPHs in a coherent-state

\footnotetext{
*Present address: Optical Nanostructures Laboratory, Columbia University, New York, NY, 10027.
}

background $[1,4]$, which do not have direct equivalences with entangled photon-pair states.

In Sec. II, we overview the basic idea of time-bin EPHs and transition from a general coherent-state background to a background formed formed by single-photon states. In Sec. III we describe a proof-of-principle experimental demonstration of time-bin EPHs in single-photon background using a conventional parametric down-conversion (PDC) photon-pair source [13] and techniques from the linear optics quantum computing toolbox [14]. Here the time-bin EPH states are sent into a "Franson interferometer" [7] and used to violate a Bell-type inequality [15]. In Sec. IV we summarize and discuss further applications of EPH states.

\section{OVERVIEW}

Perhaps the easiest way to visualize EPH states is by contrasting them with well-known photon-pair states produced by PDC. The original proposal for energy-time EPHs used two-photon absorption as a nonlinearity which simultaneously removes one photon from each of two coherent states [1]. In some sense, this is the direct opposite of continuous-wave (cw) pumped PDC, where a $\chi^{(2)}$ nonlinearity is used to simultaneously create one photon in each of two vacuum states [16]. In both cases, the creation or removal of the two photons occurs at the same time, but that time is completely uncertain, which is essentially the origin of the energy-time entanglement in these states [7].

In this way, EPHs can be thought of as the "negative image" of PDC [2]. Yet this analogy is not complete because the idea of a "single hole" (in contrast to a single PDC photon) is meaningless, and there can be many "missing pairs" in an EPH state, whereas an idealized PDC state contains just one photon-pair. Consequently, a better analogy is drawn by contrasting the relevant two-photon amplitudes involved in PDC vs EPH states [1].

To illustrate this analogy, Fig. 1 shows two different forms of two-photon amplitudes (i.e., the biphoton picture [17]) for both energy-time PDC states and energy-time EPH states. Here the term $A_{1: 2}$ represents the probability amplitude for detecting one photon in each output mode ( 1 and 2$)$ at the same time, as function of the overall time $T$. The term $A_{1,2}$ represents the probability amplitude for detecting two photons at two 

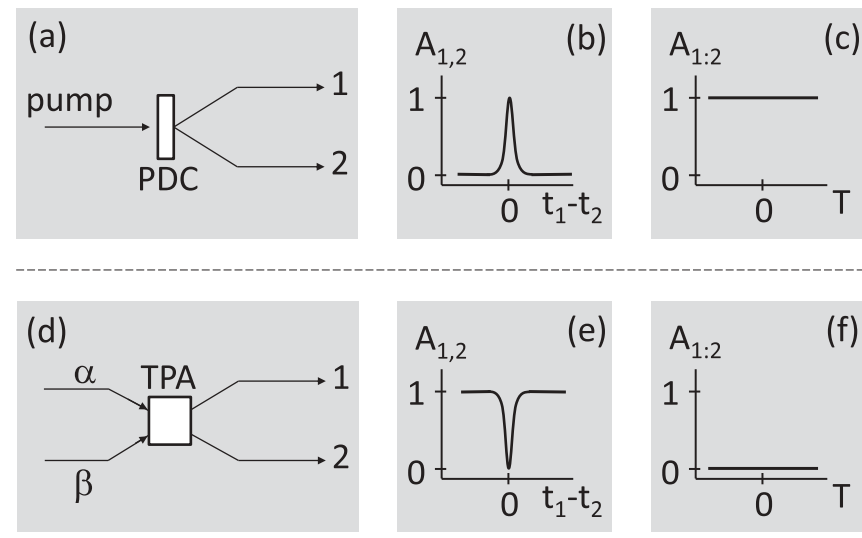

FIG. 1. Pictorial representation contrasting energy-timeentangled PDC photon-pair states [panels (a)-(c)], with energy-time EPH states [panels (d)-(f)] realized through strong two-photon absorption (TPA) [1]. By contrasting the two-photon amplitude in panel (b) vs that in panel (e), as well as that in panel (c) vs that in panel (f), it can be seen that EPHs can be thought of as the "negative image" of PDC. A description of the two kinds of two-photon amplitudes, $A_{1,2}$ and $A_{1: 2}$, is found in the main text. The amplitudes have been normalized to 1 in each plot for convenience.

different times $\left(t_{1}\right.$ and $\left.t_{2}\right)$, as a function of the difference in these times.

Figure 1(a) shows the familiar scenario for an idealized cw PDC source. Here the PDC crystal can only produce the two photons at the same time, so the amplitude $A_{1,2}$ is zero everywhere except near $t_{1}-t_{2}=0$ as shown in Fig. 1(b) (the width and shape of this peak is determined by phase-matching considerations and other details of the PDC process [16]). Due to the cw nature of the PDC pump, this photon pair can be produced at any time with equal probability, so the amplitude $A_{1: 2}$ is a constant value for all times $T$ as shown in Fig. 1(c).

In contrast, Fig. 1(d) shows the energy-time EPH scenario of Ref. [1]. Here, two narrowband weak coherent states $|\alpha\rangle$ and $|\beta\rangle$ pass through an idealized two-photon absorption (TPA) medium. The central frequencies of these coherent states are nondegenerate and chosen in such a way that the TPA medium does nothing to either beam by itself, but absorbs one photon from each state if the beams pass through at the same time [1]. Consequently, the amplitude $A_{1,2}$ is a constant value everywhere, but goes towards zero near $t_{1}-t_{2}=0$ as shown in Fig. 1(e) (the width and shape of this dip is determined by the lifetimes of the relevant states and other properties of the TPA system [18]). In the the idealized limit of infinitely strong TPA, the probability of detecting two photons at the same time is always zero, so the amplitude $A_{1: 2}=0$ for all times $T$ as shown in Fig. 1(f).

Contrasting the two-photon amplitudes of Fig. 1(b) vs Fig. 1(e) and Fig. 1(c) vs Fig. 1(f) provides a clear analogy of EPHs as the "negative image" of PDC.

We now use this analogy to introduce the idea of time-bin EPHs, which is the primary thrust of this paper. The main idea is to confine the usual cw background of Fig. 1(d) into two well-defined time bins. As in the case of Gisin et al.'s time-bin-entangled photon pairs [11,12], this is accomplished using short optical pulses and unbalanced Mach-Zehnder interferometers (MZs).
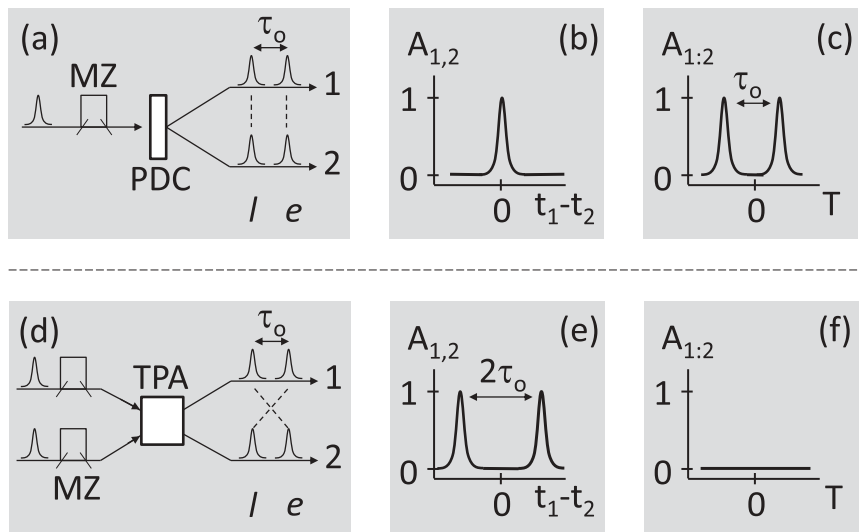

FIG. 2. Pictorial representation contrasting time-bin-entangled PDC states [panels (a)-(c)] realized through the use of a pump Mach-Zehnder interferometer (MZ) $[11,12]$ with time-bin EPH states [panels (d)-(f)]. The introduction of these time-bin EPH states is the main thrust of this paper. The optical pulses on the left side of panel (d) form the background in which the time-bin EPHs will exist. These pulses can be either coherent-state pulses or nonclassical single-photon pulses as described in the text.

Figure 2(a) first shows the standard scenario for time-binentangled photon pairs produced by pulsed PDC [11,12]. Here a short pumping pulse is divided into two coherent amplitudes by a MZ before the PDC crystal. The source produces one pair of photons in a superposition of two time bins (labeled $e$ and $l$ for "early" and "late") separated by the MZ imbalance time $\tau_{o}$. The photons will always be found in the same time bin, so again $A_{1,2}$ is only nonzero when $t_{1}-t_{2}=0$ as shown in Fig. 2(b). However, the possible pair production time is now confined to the two time bins, so $A_{1: 2}$ shows only two peaks separated by $\tau_{o}$ as shown in Fig. 2(c).

Time-bin EPH states are now constructed by designing a system that produces the negative image of Figs. 2(b) and 2(c). One conceptual method is illustrated in Fig. 2(d). Here two nondegenerate coherent-state pulses pass through two independent MZs before entering the idealized TPA medium. The response time of the TPA process is assumed to be much shorter than $\tau_{o}$, so that pairs of photons (one in each mode) in different time bins pass through without absorption. However, the possibility of two photons (one in each mode) exiting in the same time bin is removed by the TPA process. Consequently, $A_{1,2}$ shows two separated background peaks, but a missing central peak at $t_{1}-t_{2}=0$ as shown in Fig. 2(e). The TPA process also causes $A_{1: 2}=0$ in all time bins, as shown in Fig. 2(f).

Considering the relevant time bins, by contrasting the amplitudes shown in Fig. 2(b) vs Fig. 2(e) and in Fig. 2(c) vs Fig. 2(f), it can be seen that the pulsed source of Fig. 2(d) generates time-bin EPHs. It is important to emphasize that the pulses of Fig. 2(d) are thus far assumed to be coherent states, which provides time-bin EPHs in a coherent state background. This case is particularly relevant, as it is essentially the coherent-state background which allows EPH states to be amplified under certain conditions [4] and time-bin techniques are known to be generally robust in quantum systems [12]. Because the dominant sources of errors in quantum communication systems can originate from loss and 
polarization errors [19], the combination of these two ideas might offer practical benefits for future systems.

On a more fundamental level, however, it is interesting to consider replacing the coherent-state input pulses in Fig. 2(d) with single-photon input pulses. In this case, the two-photon amplitudes in Figs. 2(e) and 2(f) remain the same, but the system produces time-bin EPHs in a single-photon background. This case is particularly instructive, because a time-bin EPH state in a background formed by single-photon state becomes physically identical to one of the conventional time-bin-entangled photon-pair Bell states [11]:

$$
\left|\psi^{-}\right\rangle=\frac{1}{\sqrt{2}}\left(|l\rangle_{1}|e\rangle_{2}-|e\rangle_{1}|l\rangle_{2}\right)
$$

where the kets $|e\rangle$ and $|l\rangle$ represent, respectively, a singlephoton in the early or late time bin [20].

In other words, there are known to be exactly two photons in the system (one in each output mode), but the existence of the holes prevents them from being found in the same time bin. This appears to be the limiting case where EPH states and entangled photon-pair states reduce to the same physical state. The significance of this relatively simple scenario using both time bins and a single-photon background provides insight into the fundamental nature of EPH states in more complicated scenarios. Specific examples of these more complicated scenarios include energy-time EPH states in single-photon backgrounds and EPH states (either time bin or energy time) in coherent-state backgrounds [4].

Additionally, this unique combination of time bins and a single-photon background allows an experimental investigation of time-bin EPHs with current technology. Indeed, a direct realization of the system sketched in Fig. 2(d) (for coherent-state or single-photon backgrounds) would require strong TPA at single-photon intensities, which is exactly the type of nonlinearity needed for photonic quantum computing $[21,22]$ and is difficult with current technology. However, by considering single-photon backgrounds, proof-of-principle EPH experiments can be done by starting with two single photons and putting them in time bins in such a way as to realize the state of Eq. (1). In our experiment, we accomplish this using two photons produced from a typical cw PDC source, as described in the next section.

\section{EXPERIMENT}

A conceptual overview of our experimental demonstration of time-bin EPHs is shown in Fig. 3. The idea is to perform a Bell-inequality test by sending time-bin EPH states through a conventional Franson interferometer [7]. For simplicity, we consider a Franson arrangement using the polarization-based unbalanced MZs of Ref. [23] to eliminate the need for large path-length imbalances and fast electronics.

The EPH state of interest is generated by forming the background using two orthogonally polarized single photons (denoted by the red and blue circles labeled $|H\rangle$ and $|V\rangle$ ) separated by time $\tau_{o}$ and mixing them at a 50:50 beamsplitter. Because postselection in the output of the Franson interferometer will only register cases in which one background photon passes through each MZ, the relevant part of the two-photon state at the output of the 50:50 beamsplitter is given by Eq. (1).

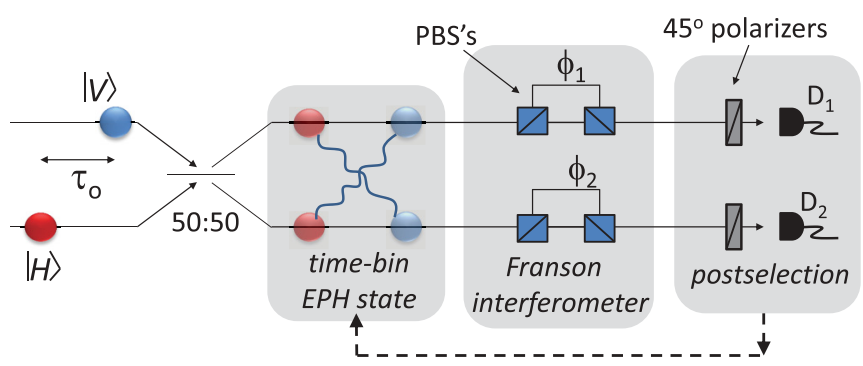

FIG. 3. (Color online) Schematic overview of our experimental method to violate Bell's inequality using time-bin EPHs in a single-photon background. The background is formed by two single photons that are displaced by $\tau_{o}$ and mixed at a 50:50 beamsplitter. Postselection at the output of a Franson interferometer realizes the time-bin EPH state in the background state given by Eq. (2). Polarization-encoding and polarizing beamsplitters (PBSs) are used to simplify the actual experiment [23].

The "early-late" and "late-early" terms of this background photon-pair state are denoted graphically by the two "linked" amplitudes in Fig. 3, while the EPH state itself is perhaps best described by the two-photon amplitudes of Figs. 2(e) and 2(f).

The polarizing beamsplitters (PBSs) direct horizontally polarized amplitudes along the short arms of the MZs and vertically polarized amplitudes along the long arms, while polarizers oriented at $45^{\circ}$ in the outputs erase any which-path information [23]. Consequently, the Franson interferometer of Fig. 3 transforms the background photon-pair state of Eq. (1) into the following state:

$$
\left|\psi^{-}\right\rangle=\frac{1}{\sqrt{2}}\left(|S\rangle_{1}|L\rangle_{2}-e^{i\left(\phi_{1}-\phi_{2}\right)}|L\rangle_{1}|S\rangle_{2}\right),
$$

where the kets $|S\rangle$ and $|L\rangle$ represent, respectively, a singlephoton in the short or long arms of the unbalanced MZs, and $\phi_{1}$ and $\phi_{2}$ are the relevant phase differences (an overall phase factor has been omitted).

When the path-length imbalances of the MZs are set to match $\tau_{o}$, the two terms in Eq. (2) are indistinguishable and lead to a coincidence counting rate between detectors $D_{1}$ and $D_{2}$ that goes like $R_{c} \sim 1+\cos \left(\phi_{1}-\phi_{2}\right)$ [13]. This " $S L$ vs $L S$ " scenario is slightly different than typical " $S S$ vs $L L$ " Franson interferometery where the coincidence counting rate goes like $R_{c} \sim 1+\cos \left(\phi_{1}+\phi_{2}\right)$ [7]. In both cases, the dependence on the difference or sum of spatially separated phases is the signature of nonlocal behavior. As in the more typical case, a visibility of this two-photon interference pattern greater than $71 \%$ can be used to violate a Bell inequality using time-bin EPHs [24].

Figure 4 shows our experimental setup used to implement the idea of Fig. 3. A type I PDC source (0.7-mm-thick BBO crystal) was pumped by a cw ultraviolet diode laser ( $\sim 40 \mathrm{~mW}$ at $407 \mathrm{~nm}, \sim 0.1-\mathrm{nm}$ linewidth). The source produced horizontally polarized photon pairs at $814 \mathrm{~nm}$ which were coupled into a 50:50 single-mode-fiber beamsplitter. Translatable glass wedges were used to introduce and adjust the time-bin separation $\tau_{o}$, and fiber polarization controllers (fpc's) on the input ports of the beamsplitter were used to prepare the states $|H\rangle$ and $|V\rangle$.

The polarization-based unbalanced MZs of Fig. 3 were implemented using two polarization-maintaining (PM) fibers 


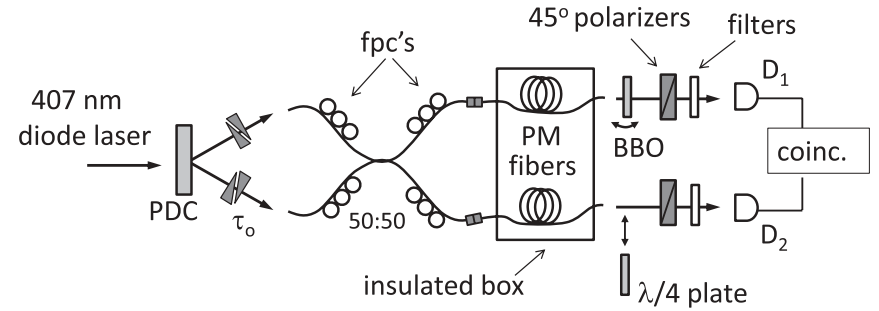

FIG. 4. Experimental apparatus used to implement the conceptual overview of Fig. 3. A PDC source provides the two background photons, and polarization-maintaining (PM) fibers are used to implement the polarization-based MZs. The phases $\phi_{1}$ and $\phi_{2}$ are controlled by an adjustable birefringent BBO slab and a quarter-wave plate as described in the text. Fiber polarization controllers (fpc's) are used to define and maintain the relevant polarization states, and 10-nm bandpass interference filters are used to define a PDC photon coherence time of $\sim 200 \mathrm{fs}$.

with their fast and slow axes oriented in the horizontal and vertical directions, respectively. The PM fibers were $2 \mathrm{~m}$ long, which gave a MZ path-length imbalance of $\tau_{o} \sim 2.6 \mathrm{ps}$ for the 814-nm photons. This imbalance greatly exceeded the coherence time of the PDC photons $(\sim 200 \mathrm{fs})$, as required in Franson interferometry [7].

The phase $\phi_{1}$ was controlled by adding a birefringent slab (in this case, a second 0.7-mm-thick BBO crystal) with its fast and slow axes aligned to match those of the PM fiber. Twisting this slab around the vertical axis changed its effective thickness and allowed smooth scanning of $\phi_{1}$. For completeness, we added or removed a quarter-wave plate at the output of PM fiber 2 for two different relative values of $\phi_{2}\left(\frac{\pi}{2}\right.$ or 0$)$.

From a technical point of view, this setup is very robust against phase drifts. Indeed, the two-photon state of Eq. (2) is insensitive to the relative phase difference between the two original photons [13]. Consequently, the delay $\tau_{o}$ introduced by the glass wedges (and set equal to that of the PM fibers) only needed to be adjusted to within the coherence length of the PDC photons, rather than interferometrically stabilized; this is similar to the case of typical Hong-Ou-Mandel experiments [25]. Additionally, by placing the relatively short PM fibers in a thermally insulated box, temperature-dependent phase drifts in the MZs were greatly reduced and active locking was not needed to stabilize $\phi_{1}$ and $\phi_{2}$.

Figure 5 shows typical experimental results obtained with this setup. The data show the expected sinusoidally varying coincidence counting rate as a function of $\phi_{1}$, for the two different relative values of $\phi_{2}\left(0\right.$ and $\left.\frac{\pi}{2}\right)$. The MZ phase difference $\phi_{1}$ could be scanned through $2 \pi$ by a very small twist of the birefringent slab $\left(\sim 1^{\circ}\right)$, so the phase was approximated as linearly changing with the twist-angle setting on a precision rotation stage. The calibration of the $x$ axis in the figure was verified by using the same scanning range and the known phase-shift of $\frac{\pi}{2}$ that exists between the two data sets (i.e., with and without a known quarter-wave plate).

The two data sets in Fig. 5 were best-fit to sinusoidal curves (constrained to a common period) with visibilities of $(86.1 \pm 0.5) \%$ and $(81.7 \pm 0.5) \%$. The deviation from an ideal $100 \%$ visibility was most likely due to standard modematching imperfections in PDC experiments, and we believe

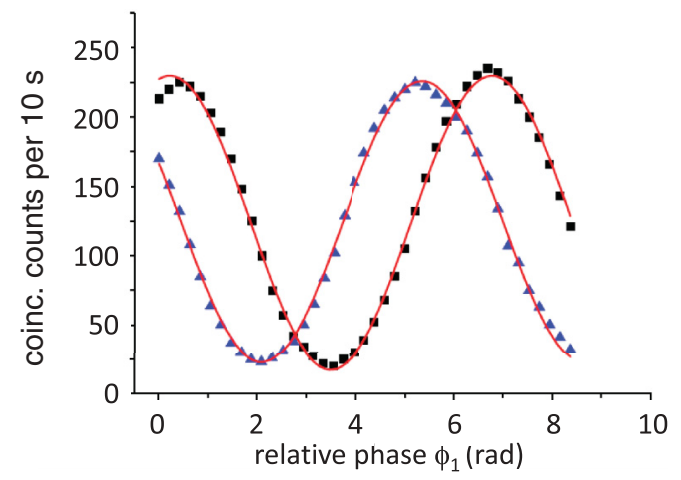

FIG. 5. (Color online) Experimental violation of Bell's inequality with time-bin EPHs. The solid lines are least-squares fits to the data constrained by a common period. The visibility of the black-squares curve is $(86.1 \pm 0.5) \%$, and the blue-triangles curve is $(81.7 \pm 0.5) \%$.

the slightly lower value for the second curve was introduced by nonideal alignment of the additional quarter-wave plate. In any event, these high visibilities (>71\% [24]) represent our proof-of-principle demonstration of a violation of Bell's inequalities using time-bin EPHs.

As a tangential side note, it is interesting to consider how the physical setup sketched in Fig. 3 can also be viewed as a timebin analog of the original Shih-Alley experiment for generating polarization entanglement via postselection [8]. In the original Shih-Alley experiment, two orthogonal polarization qubits $(|H\rangle$ and $|V\rangle)$ are mixed at a 50:50 beamsplitter and measured with polarization qubit analyzers (i.e., polarizers). In Fig. 3, two orthogonal time-bin qubits $(|e\rangle$ and $|l\rangle)$ are mixed at a 50:50 beamsplitter and measured with time-bin qubit analyzers (i.e., unbalanced $M Z$ interferometers [12]). This analogy is particularly clear if one considers the setup of Fig. 3 with no polarization dependence.

\section{SUMMARY AND DISCUSSION}

In this paper we have extended the original work on energy-time EPHs [1-3] to introduce the idea of time-bin EPHs. We have also considered the possibility of EPHs existing in backgrounds formed by single-photon states.

The initial proposal for energy-time EPHs considered cw coherent-state background beams [1], whereas the initial experiments essentially considered a discretized version with the background spread out into infinitely long coherent pulsetrains [2,3]. Here we considered the case when the EPHs exist in a coherent-state background that is confined to two well-defined time bins. When this coherent-state background is further reduced to a single-photon background, the time-bin EPH state becomes physically equivalent to a particular timebin-entangled photon-pair state [11]. We used a conventional PDC photon-pair source to generate this background and were thereby able to perform a proof-of-principle experimental violation of Bell's inequality using time-bin EPHs in a Franson-type interferometer [7].

The interpretation of any photon counting experiments related to EPHs is nonintuitive, as it is indeed the background photons which are being detected rather than any "photon holes." In Franson interferometer experiments, the simplest 
explanation is that the existence of the EPHs suppresses the coincidence counting rate in a nonlocal way. Roughly speaking, the photon holes prevent the possibility of two photons being found at the same time. This is particularly clear in the time-bin EPH states studied here, but applies to the energy-time case as well [1].

Methods to generate other forms of EPHs, such as those based on polarization, other degrees of freedom, or higherdimensional systems, remain an open question. The closely related idea of generating entanglement through superpositions of correlated phase disturbances (rather than correlated loss) has recently been proposed [26]. We hope that states of this kind, as well as the time-bin EPH states considered here, provide new insights into the nature of entanglement and valuable resources for quantum-information-processing applications.

Note added in proof. Recently, we became aware of a preprint [27] which describes a telecom-band realization of the state in Eq. (1). The authors aptly named this the "cross time-bin" entangled state and convincingly demonstrate its utility for quantum key distribution.

\section{ACKNOWLEDGMENT}

This work was supported by DARPA DSO under Grant No. W31P4Q-10-1-0018.
[1] J. D. Franson, Phys. Rev. Lett. 96, 090402 (2006).

[2] T. B. Pittman and J. D. Franson, Phys. Rev. A 74, 041801(R) (2006).

[3] I. Afek, O. Ambar, and Y. Silberberg, Phys. Rev. Lett. 105, 093603 (2010).

[4] J. D. Franson, Phys. Rev. A 84, 043831 (2011).

[5] A. K. Ekert, Phys. Rev. Lett. 67, 661 (1991).

[6] S. Rosenblum, A. Hayat, P. Ginzburg, D. Neiman, and M. Orenstein, Phys. Rev. A 81, 053848 (2010).

[7] J. D. Franson, Phys. Rev. Lett. 62, 2205 (1989).

[8] Y. H. Shih and C. O. Alley, Phys. Rev. Lett. 61, 2921 (1988).

[9] P. G. Kwiat, K. Mattle, H. Weinfurter, A. Zeilinger, A. V. Sergienko, and Y. H. Shih, Phys. Rev. Lett. 75, 4337 (1995).

[10] J. G. Rarity and P. R. Tapster, Phys. Rev. Lett. 64, 2495 (1990).

[11] J. Brendel, N. Gisin, W. Tittel, and H. Zbinden, Phys. Rev. Lett. 82, 2594 (1999).

[12] W. Tittel, J. Brendel, H. Zbinden, and N. Gisin, Phys. Rev. Lett. 84, 4737 (2000).

[13] J. Liang, Ph.D. dissertation, UMBC Publication Archives, May 2012.

[14] E. Knill, R. LaFlamme, and G. J. Milburn, Nature (London) 409, 46 (2001).
[15] J. Bell, Speakable and Unspeakable in Quantum Mechanics (Cambridge University Press, New York, 1987).

[16] D. N. Klyshko, Photons and Nonlinear Optics (Gordon \& Breach, New York, 1988).

[17] Y. H. Shih, An Introduction to Quantum Optics: Photon and Biphoton Physics (Taylor \& Francis, New York, 2011).

[18] R. W. Boyd, Nonlinear Optics (Academic Press, New York, 2008).

[19] N. Gisin, G. G. Ribordy, W. Tittel, and H. Zbinden, Rev. Mod. Phys. 74, 145 (2002).

[20] Equation (1) is the time-bin analog of the $\left|\psi^{-}\right\rangle$polarizationentangled state described, for example, in Ref. [9].

[21] G. J. Milburn, Phys. Rev. Lett. 62, 2124 (1989).

[22] J. D. Franson, B. C. Jacobs, and T. B. Pittman, Phys. Rev. A 70, 062302 (2004).

[23] D. V. Strekalov, T. B. Pittman, A. V. Sergienko, Y. H. Shih, and P. G. Kwiat, Phys. Rev. A 54, 1(R) (1996).

[24] J. F. Clauser and A. Shimony, Rep. Prog. Phys. 41, 1881 (1978).

[25] C. K. Hong, Z. Y. Ou, and L. Mandel, Phys. Rev. Lett. 59, 2044 (1987).

[26] B. T. Kirby and J. D. Franson, arXiv:1207.5487.

[27] A. Martin, F. Kaiser, A. Vernier, A. Bevartos, V. Scarani, and S. Tanzilli, arXiv:1207.6586. 